\title{
MONASHUniversity
}

Department of Econometrics and Business Statistics

http://www.buseco.monash.edu.au/depts/ebs/pubs/wpapers/

\section{Rainbow plots, bagplots and boxplots for functional data}

Rob J Hyndman and Han Lin Shang

November 2008

Working Paper 09/08 


\section{Rainbow plots, bagplots and boxplots for functional data}

\section{Rob J Hyndman}

Department of Econometrics and Business Statistics,

Monash University, VIC 3800

Australia.

Phone number: 61-3-99055141

Email: Rob.Hyndman@buseco.monash.edu

\section{Han Lin Shang}

Department of Econometrics and Business Statistics, Monash University, VIC 3800

Australia.

Phone number: 61-3-99059280

Email: HanLin.Shang@buseco.monash.edu

9 November 2008

JEL classification: $\mathrm{C} 14, \mathrm{C} 80$ 


\title{
Rainbow plots, bagplots and boxplots for functional data
}

\begin{abstract}
We propose new tools for visualizing large numbers of functional data in the form of smooth curves or surfaces. The proposed tools include functional versions of the bagplot and boxplot, and make use of the first two robust principal component scores, Tukey's data depth and highest density regions.

By-products of our graphical displays are outlier detection methods for functional data. We compare these new outlier detection methods with existing methods for detecting outliers in functional data and show that our methods are better able to identify the outliers.
\end{abstract}

Keywords: Highest density regions, Robust principal component analysis, Kernel density estimation, Outlier detection, Tukey's halfspace depth. 


\section{Introduction}

Functional data are becoming increasingly common in diverse fields, and there is a need to develop new statistical tools for analyzing such data. In this paper, we are interested in visualizing data comprising smooth curves or surfaces (e.g., Ramsay \& Silverman 2005, Locantore et al. 1999). Such functional data may be age-specific mortality or fertility rates (Hyndman \& Ullah 2007), term-structured yield curves (Kargin \& Onatski 2008), spectrometry data (Reiss \& Ogden 2007), or one of the many applications described by Ramsay \& Silverman (2002). Ramsay \& Silverman (2005) and Ferraty \& Vieu (2006) provide detailed surveys of the many parametric and nonparametric techniques for analyzing functional data.

Visualization methods help in the discovery of characteristics that might not have been apparent with mathematical models and summary statistics; yet this area of research has not received much attention in the functional data analysis literature to date. Most of the literature focuses on the modelling, clustering and forecasting of functional data, with visualization playing, at best, a minor role. Notable exceptions are the phase-plane plot of Ramsay \& Ramsey (2002) and the rug plot of Hyde et al. (2006), which highlight important distributional characteristics from the first and second derivatives of functional data. Another exception is the singular value decomposition plot of Zhang et al. (2007) which displays the changes in latent components as sample size or dimensionality increases. We aim to contribute to the functional data analytic toolbox by proposing three new graphical methods: the rainbow plot, the functional bagplot and the functional highest density region (HDR) boxplot.

A side benefit of two of these new graphical methods is the identification of outliers, which may not be obvious from a plot of the original data. Outlying curves may lie outside the range of the vast majority of the data (we call these "magnitude outliers"), or they may be within the range of the rest of the data but have a very different shape from other curves (we call these "shape outliers"), or they may exhibit a combination of these features. Any attempt to identify outlying curves should be able to deal with all types of outliers. 
The presence of outliers has a serious effect on the modelling of functional data. Statistical analysis without identifying outliers can often lead to inaccurate conclusions. Despite the obvious importance of this problem, we are aware of only two previous approaches to functional outlier detection. Hyndman \& Ullah (2007) used a method based on robust principal component analysis, while Febrero et al. $(2007,2008)$ considered functional outlier detection using successive likelihood ratio tests and smoothed bootstrapping.

Suppose we have a set of curves or surfaces $\left\{y_{i}(x)\right\}, i=1, \ldots, n$. We are interested in visualizing these curves or surfaces for large $n$, and we are interested in identifying outliers in the observed data. From now on, we will only refer to "curves" rather than "curves or surfaces" and we will assume $x$ is scalar. However, the results that follow are easily generalized to $x$ of higher dimension.

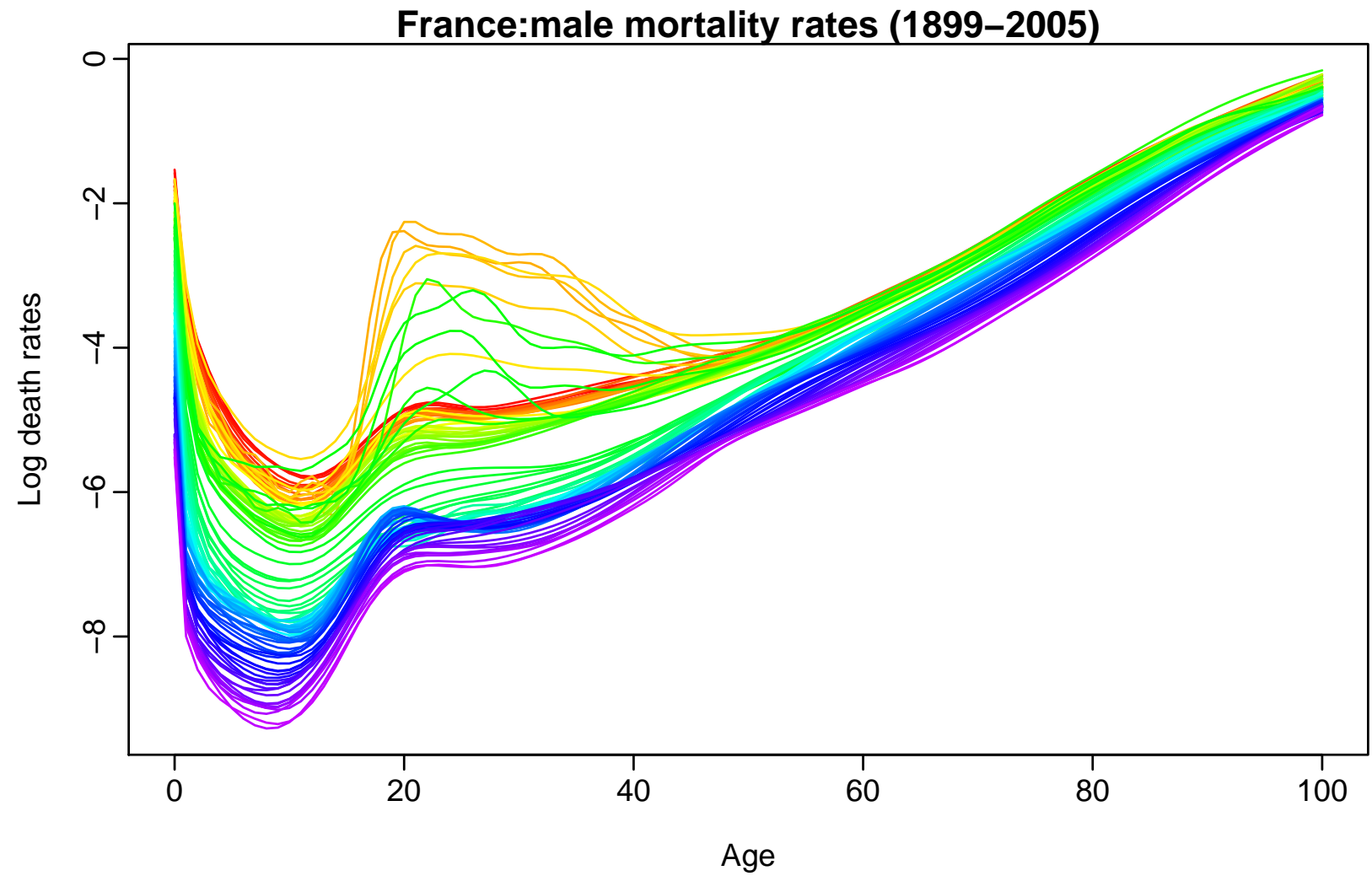

Figure 1: French male age-specific mortality rates (1899-2003). The oldest curves are shown in red, with the most recent curves in violet. Curves are ordered chronologically according to the colors of the rainbow. 
To motivate the discussion, consider Figure 1 showing annual smoothed age-specific mortality curves for French males between 1899 and 2003. The data were taken from the Human Mortality Database (2008), and smoothed using penalized splines as described in Hyndman \& Ullah (2007). The mortality rates are the ratios of death counts to population exposure in the relevant year for the given age (based on one-year age groups). In this example, $y_{i}(x)$ denotes the logarithm of the mortality rates in year $i$ for males of age $x$. Outliers clearly exist in the data due to wars and epidemics, and we seek to identify them.

Figure 1 is an example of a "rainbow plot" where the colors of the curves follow the order of a rainbow with the oldest data in red and the most recent data in violet. This is one of the plots discussed in Section 3.

In Figure 1, some mortality curves shown in yellow and green colors indicate sudden increases in mortality rates between ages 20 and 40 for a number of years. These are due to dramatic changes in mortality patterns resulting from the first and second World Wars, as well as the Spanish flu which occurred in 1918 and 1919.

With a large number of overlapping curves, it is difficult to identify where the "median curve" might lie, or where the bulk of the data fall. It may also be difficult to see outliers if they are obscured by other curves (for example, curves having a different shape from the rest of the data). With univariate data, we commonly use boxplots to solve these issues. So, we aim to define functional variations on boxplots which will show outlying curves, a "central" curve, and a region containing the "middle" $50 \%$ of curves.

The rainbow plot is a simple plot of all the data, with the only added feature being a color palette based on an ordering of the data. Figure 1 shows time-ordering, but other possibilities are based on data depth, data density or some other unique ranking procedures. In Section 2, we explore various ordering methods for functional data and show how the rainbow plot can be surprisingly illuminating with a careful choice of ordering. 
Two of the ordering methods that will prove useful are based on a robust principal component algorithm applied to the first two principal component scores calculated from $\left\{y_{i}(x)\right\}$. These bivariate points can then be ordered, for example, by applying Tukey's (1974) halfspace depth. This idea immediately lends itself to a functional bagplot obtained by applying the bivariate bagplot (Rousseeuw et al. 1999) to the first two principal component scores. Recently, this idea has been implemented to cluster functional data in Sood et al. (2009).

Similarly, a functional HDR boxplot is defined by computing a bivariate kernel density estimate (Scott 1992) on the first two principal component scores, and then applying the bivariate HDR boxplot of Hyndman (1996). The HDR boxplot has the advantage of being able to display multimodality if it is presented in the data. The bagplot and boxplot are introduced in Section 3.

Outliers in the functional data are identified as outliers in the bivariate score space. A related idea has previously been employed by Jones \& Rice (1992) for solving the graphical obscurity problem associated with large collections of curves. The outlier detection methods made possible by our functional bagplot and functional boxplot are compared in Section 4 with several existing functional outlier detection methods and multivariate outlier detection methods applied to the discretized functions.

Section 5 provides a summary of our main results, and some thoughts on how the plots might be extended.

\section{Ordering functional data}

All of our graphical methods involve some kind of ordering of the functional data. Figure 1 showed the data in time-order, but for many data sets we will want an ordering based on the values of the data themselves. In this section, we review some possible ordering methods, and consider how they can be used in conjunction with the rainbow plot. 
Each of the ordering methods use a form of data depth (Tukey 1974), which provides a way to measure the "depth" or "outlyingness" of a given observation with respect to the set of observations or their underlying distribution. Often, contours of a depth function are used to reveal the shape and structure of multivariate data. Recent studies of data depth include Rousseeuw \& Ruts (1998, 1999), Rousseeuw et al. (1999), Struyf \& Rousseeuw (2000) and Wang \& Serfling (2006). A seminal study in data depth can be found in Liu et al. (1999) who listed variants of data depth, while Zuo \& Serfling (2000) investigated four properties of data depth.

\subsection{Functional depth method}

Febrero et al. (2007) proposed an outlier detection method that used the notion of functional depth defined as

$$
o_{i}=\int D\left(y_{i}(x)\right) d x
$$

where $D\left(y_{i}(x)\right)$ is a univariate depth measure for a specific value of $x$. Using this definition, we define the ordering of the curves by the increasing order of $\left\{o_{i}\right\}$, so the first curve has the lowest functional depth and the last curve has the greatest functional depth.

However, as the functional depth is calculated by integrating univariate depth, this method may not detect curves that have an unusual shape but lie within the range of the majority of curves (López-Pintado \& Romo 2006). Consequently, this definition of functional depth is not adequate for much functional data. Instead, we propose a measure of functional depth based on the first two principal component scores.

\subsection{Bivariate score depth}

Let $\left\{\phi_{k}(x)\right\}$ represent the principal components and $\left\{z_{i, k}\right\}$ the principal component scores from a functional principal component decomposition. Much of the information inherent in the original data $\left\{y_{i}(x)\right\}$ is captured in the first few principal components and scores (Jones \& Rice 1992, 
Sood et al. 2009). So we will take the first two score vectors $\left(z_{1,1}, \ldots, z_{n, 1}\right)$ and $\left(z_{1,2}, \ldots, z_{n, 2}\right)$ and consider methods of bivariate depth that could be applied to these vectors. We shall refer to the bivariate point $\left(z_{i, 1}, z_{i, 2}\right)$ as $z_{i}$.

Because principal component decomposition is not resistant to outliers, we apply Croux \& RuizGazen's (2005) robust principal component algorithm which uses a form of projection pursuit. This algorithm was designed for multivariate rather than functional data, but we can apply it to discretized curves $\left\{y_{i}(x)\right\}$. The virtue of this approach is that it can still be applied even when the number of variables is significantly greater than the number of observations, which is the case with finely discretized curves.

The bivariate scores can then be ordered using Tukey's halfspace location depth (Tukey 1974), denoted by $d(\boldsymbol{\theta}, \boldsymbol{Z})$ for some point $\boldsymbol{\theta} \in R^{2}$ relative to the bivariate data cloud $\boldsymbol{Z}=\left\{\boldsymbol{z}_{i} ; i=\right.$ $1, \ldots, n\}$. Tukey's depth is defined as the smallest number of data points contained in a closed half-plane containing $\boldsymbol{\theta}$ on its boundary. Then the observations can be ordered as the distances $o_{i}=d\left(z_{i}, \boldsymbol{Z}\right)$ in increasing order. The first curve by this ordering can be considered a "median" curve, while the last curve can be considered the outermost curve in a sample of curves.

\subsection{Data density}

A third way to order the points is by the value of a kernel bivariate density estimate (Scott 1992) at each observation. Let $o_{i}=\hat{f}\left(z_{i}\right)$ where $\hat{f}(z)$ is a bivariate kernel density estimate calculated from all the bivariate principal component scores. Then the functional data are ordered by values of $\left\{o_{i}\right\}$ in decreasing order. So the curve with bivariate scores closest having highest density is the first observation and the last curve has the lowest density value. Thus, the first curve may be considered the "modal curve" while the last curve may be considered the most unusual curve.

Note that the last curve by this ordering may not take values very different from the others and its bivariate scores may not be on the edge of the scatterplot of $\left(z_{1, i}, z_{2, i}\right)$. It is possible to have a 
point on the interior of this scatterplot, but with no other points nearby, and in this case it will have low density value.

\section{Three functional graphical tools}

\subsection{Rainbow plots}

For data that are not naturally ordered by time, or by some other indexes, the rainbow plot can still be used by constructing an ordering index such as the data depth or data density indexes defined above. Then the colors are chosen in rainbow order according to the ordering of $\left\{o_{i}\right\}$.

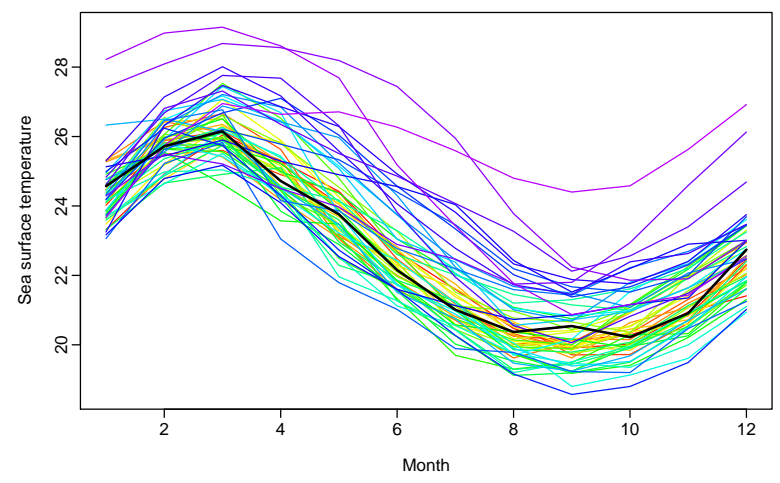

(a) Rainbow plot with depth ordering.

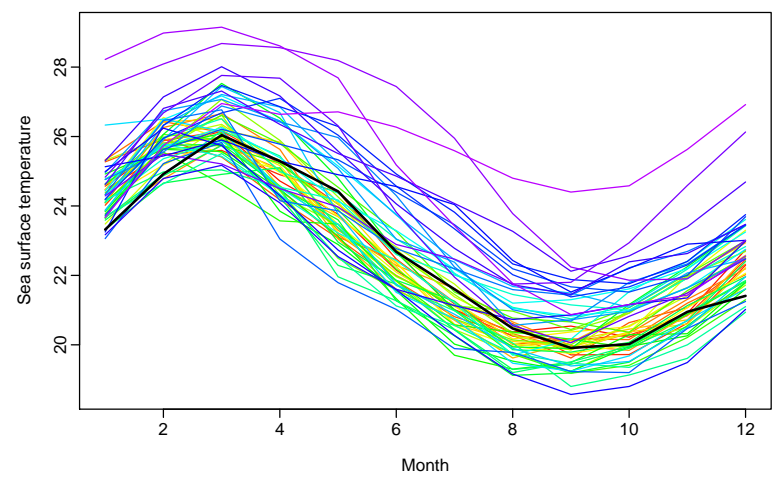

(b) Rainbow plot with density ordering.

Figure 2: Rainbow plots using different order indexes. The black lines show the median curve in (a) and modal curve in (b).

To demonstrate this, we consider a time series of average monthly sea surface temperatures from January 1951 to December 2007, available online at www.cpc.ncep.noaa.gov/data/indices. These temperatures are measured by moored buoys in the "Niño region" defined by the coordinate $0-10^{\circ}$ South and $90-80^{\circ}$ West. There is no time trend in these data, so a rainbow plot with time ordering is not particularly informative. Rainbow plots using depth and density order indexes are shown in Figure 2. The colors reflect the ordering and follow the order of the rainbow, with the curves closest to the center of the data set shown in red, while the most outlying curves are shown in violet. We plot the curves in order of depth or density, so the red curves are 
mostly obscured, but the violet outlier curves are clearly seen even if they overlap the majority of the data.

\subsection{Functional bagplot}

The functional bagplot is based on the bivariate bagplot of Rousseeuw et al. (1999) applied to the first two principal component scores. It uses Tukey's (1974) halfspace location depths. The depth region $D_{k}$ is the set of all $\boldsymbol{\theta}$ with $d(\boldsymbol{\theta}, \boldsymbol{z}) \geq k$. Since the depth regions form a series of convex hulls, we have $D_{k_{1}} \subset D_{k_{2}}$ for $k_{2}>k_{1}$. The Tukey bivariate depth median is defined as the value of $\boldsymbol{\theta}$ which minimizes $d(\boldsymbol{\theta}, \boldsymbol{Z})$ if there is a unique such $\boldsymbol{\theta}$; otherwise it is defined as the center of gravity of the deepest region.

Like a univariate boxplot, the bivariate bagplot has a central point (the Tukey median), an inner region (the 'bag') and an outer region, beyond which outliers are shown as individual points. The bag is defined as the smallest depth region containing at least $50 \%$ of the total number of observations. The outer region (or 'fence') of the bagplot is the convex hull of the points contained within the region obtained by inflating the bag (relative to the Tukey median) by a factor $\rho$. Rousseeuw et al. (1999) used a value of $\rho=3$. However, we prefer $\rho=2.58$ as that will allow the fence to contain $99 \%$ of the observations when the data follow standard normal distributions. A proof of this result is given in the Appendix.

The functional bagplot is a mapping of the bagplot of the first two robust principal component scores to the functional curves. The functional bagplot displays the median curve (the curve with greatest depth), and inner and outer regions. The inner region is defined as the region bounded by all curves corresponding to points in the bivariate bag. Thus there are $50 \%$ of curves in the inner region. The outer region is similarly defined as the region bounded by all curves corresponding to points within the bivariate fence region. 

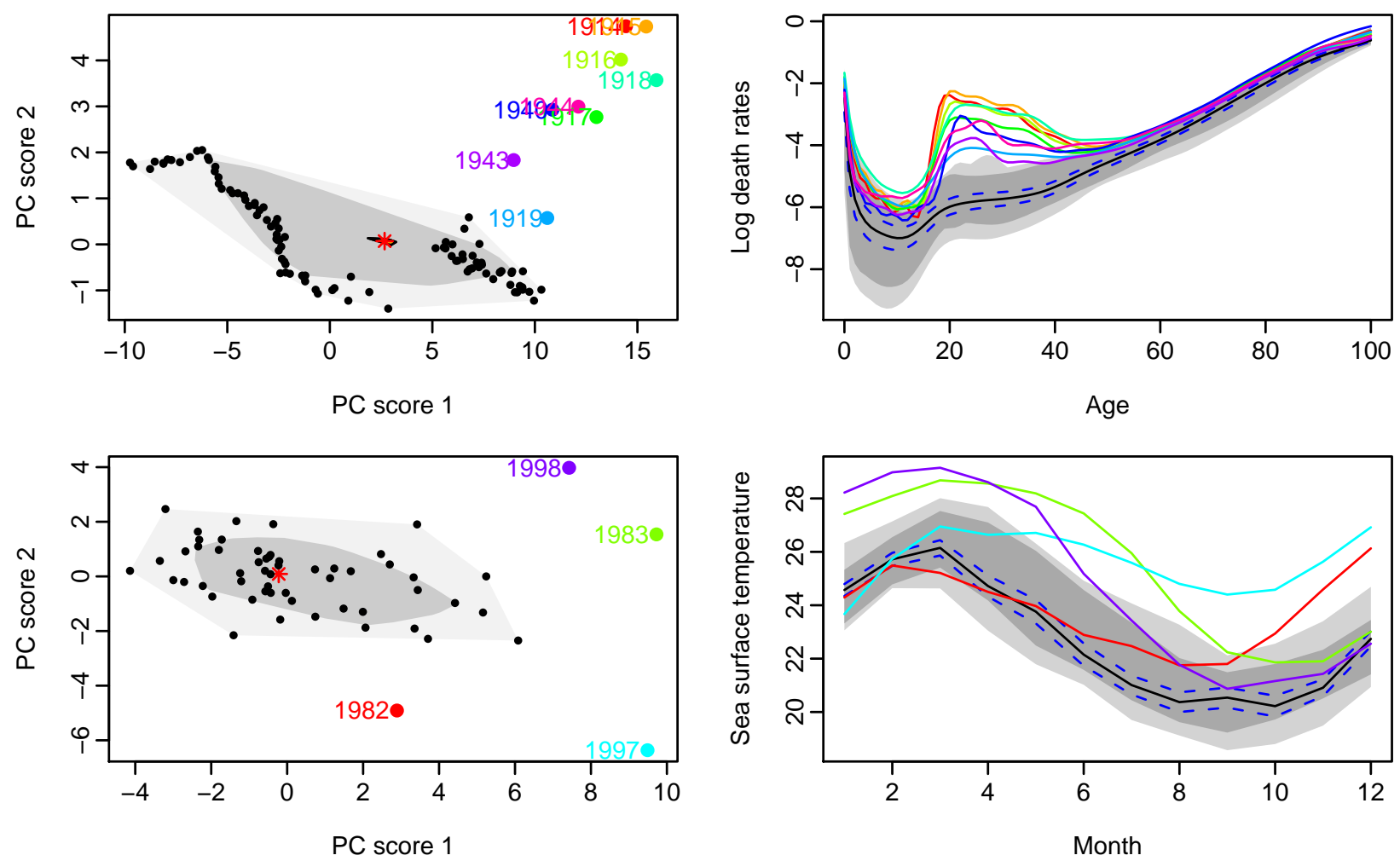

Figure 3: Bivariate bagplot and functional bagplot for the French male mortality rates (top) and the El Niño indexes (bottom). The dark and light grey regions show the bag and fence regions respectively. The red asterisk is the Tukey depth median. In the right panels, the black line is the median curve surrounded by 95\% pointwise confidence intervals. The curves outside the outer region are shown as outliers of different colors.

Two examples are shown in Figure 3 using the French male mortality data and the El Niño indexes. In the left panels, the dark grey region shows the $50 \%$ bag and the light grey region exhibits the $99 \%$ fence. These convex hulls correspond directly with the regions of similar shading in the functional bagplot on the right. Points outside these regions are identified as outliers. The different colors for these outliers enable the individual functional curves on the right to be matched to the bivariate principal component scores on the left. The red asterisk marks the Tukey median of the bivariate principal component scores, and the solid black curve in each right panel shows the median curve. The dotted blue lines in the right panels give 95\% pointwise confidence intervals for the median curve (similar to the notched boxplot of Tukey (1977)). 
The detected outliers in the French male mortality data are years 1914-1919, 1940, 1943-1944. They correspond to the first and second World Wars and the Spanish flu pandemic. The detected outliers in the El Niño data are years 1982-1983 and 1997-1998. The El Niño indexes during 1982-1983 began in June 1982 with a weak heating, then an extreme abnormal increase of sea surface temperature between September 1982 and June 1983 (Timmermann et al. 1999, Moran et al. 2006). The El Niño indexes during 1997-1998 were also unusual, especially in March and April. Dioses et al. (2002) reported that the northern central region of Peru was strongly affected as warm waters with low salinity approached the coast, while the southern region of Peru was more influenced by oceanic waters.

The functional bagplot may be a good outlier detection method when outliers are far away from the median. However, when outliers are near the median, this depth-measure outlier detection tool can misidentify outliers as shown in Section 4.5 via a simulated data set. In this situation, the functional HDR boxplot is more appropriate.

\subsection{Functional HDR boxplot}

The functional HDR boxplot is based on the bivarate HDR boxplot of Hyndman (1996) applied to the first two principal component scores. The bivariate HDR boxplot is constructed using a bivariate kernel density estimate $\hat{f}(\boldsymbol{z})$ defined as

$$
\hat{f}(z)=\frac{1}{n} \sum_{i=1}^{n} K_{h_{i}}\left(z-Z_{i}\right),
$$

where $Z_{i}$ represents a set of bivariate points, $K_{h_{i}}(\cdot)=K\left(\cdot / h_{i}\right) / h_{i}, K$ is called the kernel function and $h_{i}$ is the bandwidth for the $i$ th dimension. We select the bandwidths using smoothed cross validation (Duong \& Hazelton 2005). 
Using the kernel density estimates, a HDR is defined as

$$
R_{\alpha}=\left\{z: \hat{f}(z) \geq f_{\alpha}\right\}
$$

where $f_{\alpha}$ is such that $\int_{R_{\alpha}} \hat{f}(z) d z=1-\alpha$. That is, it is the region with probability coverage $1-\alpha$, where every point within the region has a higher density estimate than every point outside the region-hence the name "highest density region". For a bivariate density, the HDRs can be considered as contours with expanding coverage as $\alpha$ decreases.

The bivariate HDR boxplot displays the mode, defined as $\arg \sup \hat{f}(z)$, along with the 50\% inner and (usually) 99\% highest density regions. All points excluded from the outer HDR are outliers.

The functional HDR boxplot is a mapping of the bivariate HDR boxplot of the first two robust principal component scores to the functional curves. The functional HDR boxplot displays the modal curve (the curve with highest density), and inner and outer regions. The inner region is defined as the region bounded by all curves corresponding to points inside the $50 \%$ bivariate HDR. Thus there are $50 \%$ of curves in the inner region. The outer region is similarly defined as the region bounded by all curves corresponding to points within the outer bivariate HDR.

Two examples are shown in Figure 4 using the French male mortality data and the El Niño indexes. In the left panel, the dark and light grey regions show the 50\% HDR and the outer HDR respectively. These correspond directly with the regions of similar shading in the functional HDR boxplot on the right. Points outside these outer regions are identified as outliers. The use of different colors for these outliers enable the individual curves on the right to be matched to the bivariate score HDR on the left. The red dot in the left panel marks the mode of bivariate principal component scores and corresponds to the solid black curve in the right panel.

As with any outlier detection method including bagplot and HDR boxplot, the coverage probability of the outer region needs to be pre-specified. In the French male mortality data, we set the coverage probability of the outer region to be $92 \%$ while the coverage probability was set to 

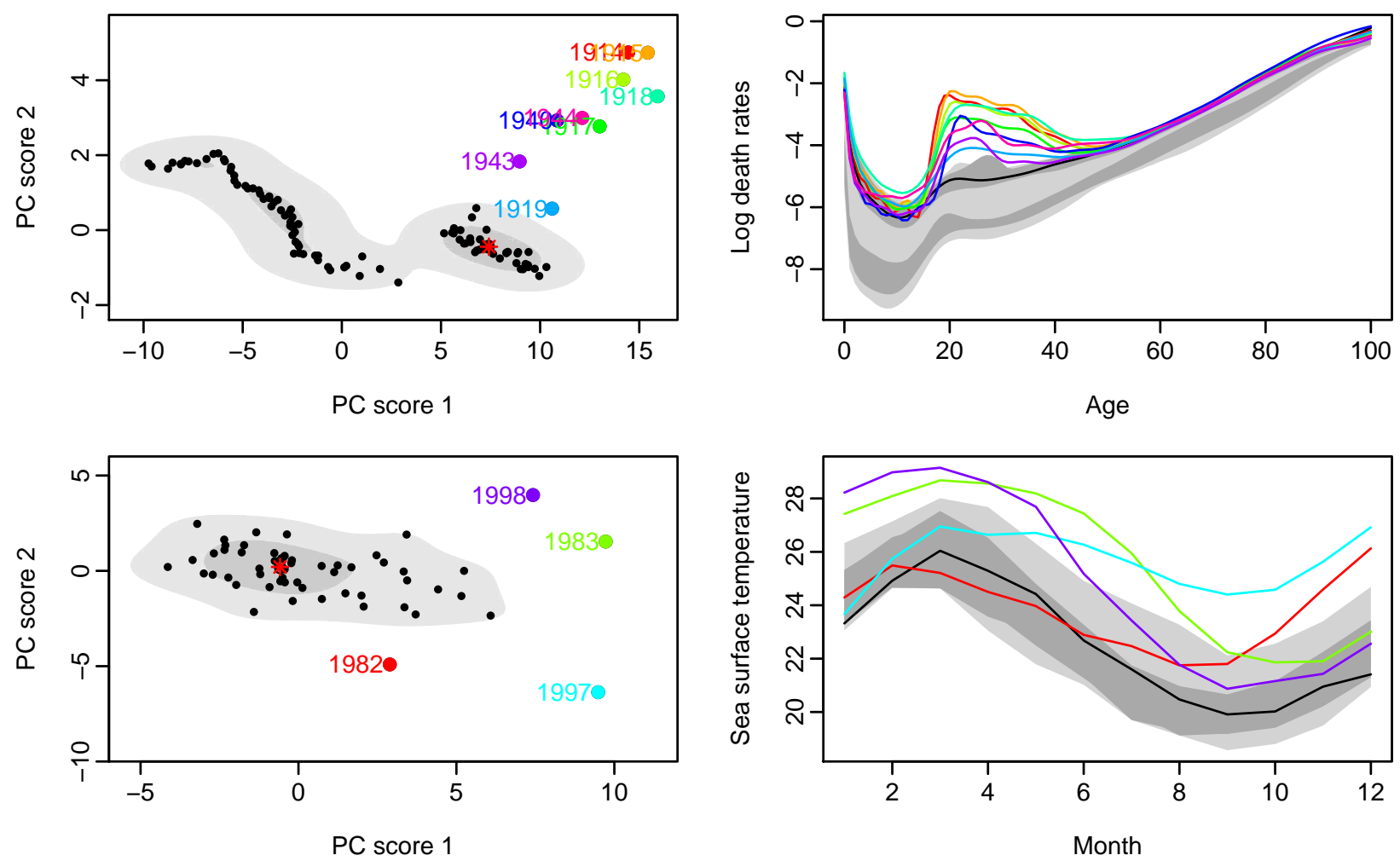

Figure 4: Bivariate HDR boxplot and functional HDR boxplot for the French male mortality rates (top) and the El Niño indexes (bottom). The dark and light grey regions show 50\% and 92\% HDR respectively. The black line is the modal curve. The curves outside the outer region are outliers.

be $93 \%$ in the El Niño data. The detected outliers in both examples match to the same results obtained by the bagplot. This indicates that those outliers have different magnitude and shape.

The presence of bimodality is seen in the top row of Figure 4. This indicates that samples may come from two populations with different locations. Further investigation shows that the two regions correspond to years before and after the end of World War II. Immediately after the war, there was a large drop in mortality rates which can be clearly seen in Figure 1.

\section{Outlier detection methods}

The functional bagplot and functional HDR boxplot identify outliers in the functional data. In this section we compare these outlier identification methods with other published methods. 


\subsection{Functional depth method}

Febrero et al. (2007) proposed an outlier detection method that calculates a likelihood ratio test statistic for each curve $y_{i}(x)$. An outlier is determined if the maximum of the test statistics is larger than a critical value $c$. This outlier is then omitted and the remaining data are tested for another outlier. The procedure continues until no more outliers can be found. The test is based on the functional depth given by (1), and so is not sensitive to shape outliers.

\subsection{Integrated squared error method}

Hyndman \& Ullah (2007) proposed an outlier detection method which utilizes robust functional principal component analysis. Let the integrated squared error for observation $i$ be

$$
v_{i}(x)=\int_{x} e_{i}^{2}(x) d x=\int_{x}\left(y_{i}(x)-\sum_{k=1}^{K} z_{i, k} \phi_{k}(x)\right)^{2} d x
$$

where $K$ is a pre-specified number of components (usually 2), $\left\{\phi_{k}(x)\right\}$ are the principal components functions and $z_{i, k}$ are their associated scores. This gives a measure of accuracy of the principal component approximation for observation $i$. High integrated squared errors indicate a high likelihood of being detected as outliers.

If $e_{i}(x)$ is normally distributed, then $v_{i}(x)$ follows a $\chi^{2}$ distribution with $\mathrm{E}\left(v_{i}(x)\right)=0.5 \operatorname{Var}\left(v_{i}(x)\right)$. Then, the probability that $v_{i}<c$, where $c=s+\lambda \sqrt{s}$ and $s=\operatorname{median}\left(\left\{v_{1}, \ldots, v_{n}\right\}\right)$, is approximately $\Phi(\lambda / \sqrt{2})$, where $\Phi(\cdot)$ is the distribution function of a standard normal distribution. For example, with $\lambda=3.29$, $\Phi(3.29 / \sqrt{2})=99 \%$.

\subsection{Robust Mahalanobis distances}

The robust Mahalanobis distance is a well-known multivariate outlier detection method, which we can apply to discretized curves $\left\{y_{i}\left(x_{j}\right) ; j=1, \ldots, p\right\}$. Assuming functional data are observed 
at $p$ equally spaced dense grid, the squared robust Mahalanobis distance is defined by

$$
r_{i}=\left(y_{i}\left(x_{j}\right)-\hat{\mu}\left(x_{j}\right)\right)^{\prime} \hat{\Sigma}^{-1}\left(y_{i}\left(x_{j}\right)-\hat{\mu}\left(x_{j}\right)\right), \quad j=1, \ldots, p,
$$

where $\hat{\mu}\left(x_{j}\right)$ is the sample mean and $\hat{\Sigma}$ is a robust estimate of the covariance matrix of $\left\{y_{i}\left(x_{j}\right)\right\}$. We assume that $\hat{\Sigma}$ is positive definite so that $\hat{\Sigma}^{-1}$ is nonsingular. The resultant distances are compared to a critical value following a $\chi^{2}$ distribution with $p$ degrees of freedom. For a predefined $\alpha=99 \%$ level, outliers are observations that have Mahalanobis squared distances greater than the critical value $\chi_{0.99, p}^{2}$. See Becker \& Gather (2001) and Hardin \& Rocke (2005) for discussion on variations of this method.

\subsection{Location-scale method}

Another multivariate outlier detection method is the location-scale approach of Filzmoser et al. (2008). This approach begins by robustly scaling the $p$ equally-spaced discretized functions by the pointwise median and median absolute deviation. They applied a robust principal component analysis and retained the number of principal components that can explain at least $99 \%$ of the total variation. Having robustly scaled the retained principal components, they calculated the robust Mahalanobis distance for each curve. They ordered the robust Mahalanobis distances by Rocke's (1996) translated biweight function, and assigned the weight $w_{1, i}$ to each observation. They repeated the above steps with a kurtosis weighted principal component analysis and obtained weight $w_{2, i}$. Outliers are detected when the weights $w_{1, i}$ and $w_{2, i}$ are both zero.

\subsection{Outlier detection performance comparison}

We applied the various outlier detection methods discussed above to the French male mortality data. Table 1 presents the comparative results and the relative computing times (using a Pentium 4 CPU 3.20GHz, 512MB of RAM). 


\begin{tabular}{lll}
\hline Method & Detected outliers & Time \\
\hline Functional depth & None & 18.83 \\
Integrated squared error & $1914-1918,1940,1943-1945$ & 3.41 \\
Functional bagplot & $1914-1919,1940,1943-1944$ & 0.30 \\
Functional HDR boxplot & $1914-1919,1940,1943-1944$ & 0.04 \\
Location-scale & $1914-1918,1940,1943-1944,1953$, & 0.09 \\
& $1960,1992-2003$ & \\
Robust Mahalanobis distance & $1914-1918,1940,1943-1944$ & 1.42 \\
\hline
\end{tabular}

Table 1: Comparison of outlier detection performance for the various methods applied to the French male mortality data.

Based on historical information, we suspect that the functional outliers would be the time periods of the first and second World Wars (1914-1918 and 1939-1945) and the Spanish flu epidemic of 1918-1919. These factors have affected the mortality pattern significantly, which provides an explanation for the sudden increases in mortality rates between ages 20 and 40 . Clearly, the functional depth method has failed to detect any of these outliers, and the location-scale method has incorrectly detected a large number of years that were not outliers. The remaining methods all do quite well at spotting the outliers.

\begin{tabular}{lll}
\hline Method & Detected outliers & Time \\
\hline Functional depth & 1983,1997 & 15.7 \\
Integrated squared error & $1951,1983,1997$ & 0.85 \\
Functional bagplot & $1982-1983,1997-1998$ & 0.33 \\
Functional HDR boxplot & $1982-1983,1997-1998$ & 0.02 \\
Location-scale & $1968,1972-1973,1982-1983,1987$, & 0.05 \\
& $1992,1997-1998,2007$ & \\
Robust Mahalanobis distance & 1983,1997 & 8.00 \\
\hline
\end{tabular}

Table 2: Comparison of outlier detection performance for the various methods applied to the El Niño indexes.

As discussed earlier, the expected outliers in the El Niño data set are years 1982-1983 and 19971998. The functional depth method, integrated squared error and robust Mahalanobis distance have failed to detect some outliers, and the location-scale method has incorrectly detected too many years that were not outliers. The functional bagplot and functional HDR boxplot do quite well at spotting the outliers. 
In these illustrations, the functional depth approach performed the worst among all methods in terms of accuracy and computing speed. This accords with the analysis of López-Pintado \& Romo (2006), who inferred that the functional depth approach does not take shape outliers into account and could potentially result in false detection. In contrast, the functional bagplot and functional HDR boxplot achieve the highest outlier detection accuracy, followed by the locationscale method, robust Mahalanobis distance approach, and the integrated squared error method.

The location-scale method tends to identify far more outliers than actually exist. The integrated squared error method depends on the number of components used in the principal component approximation, which makes it rather too subjective for regular use; it is also computationally slow because the integrated sum squared error has to be calculated for each curve. In addition, this approach often fails to detect outliers. The robust Mahalanobis distance approach is also likely to identify too few outliers, as was shown by Filzmoser (2004).

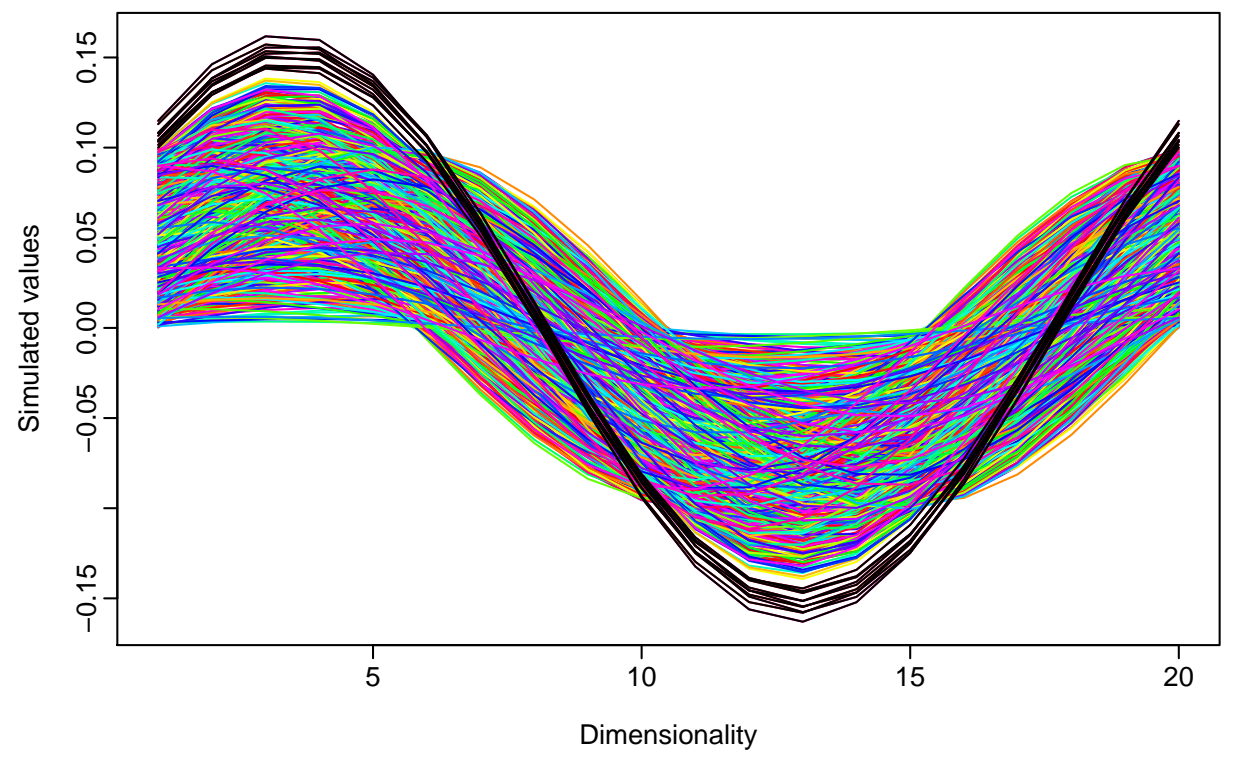

Figure 5: 1000 simulated functional curves with a functional form of $y_{i}(x)=a_{i} \sin (x)+b_{i} \cos (x)$. The 10 curves in black are outliers.

As a third example, we simulated 990 curves of the form $y_{i}(x)=a_{i} \sin (x)+b_{i} \cos (x)$, where $0<x<2 \pi$, and $a_{i}$ and $b_{i}$ follow independent uniform distributions with limits 0.0 and 0.1 . An additional ten curves were randomly simulated with the same functional form, but with $a_{i}$ and 
$b_{i}$ following uniform distributions with limits 0.1 and 0.12 . The simulated data are shown in Figure 5.
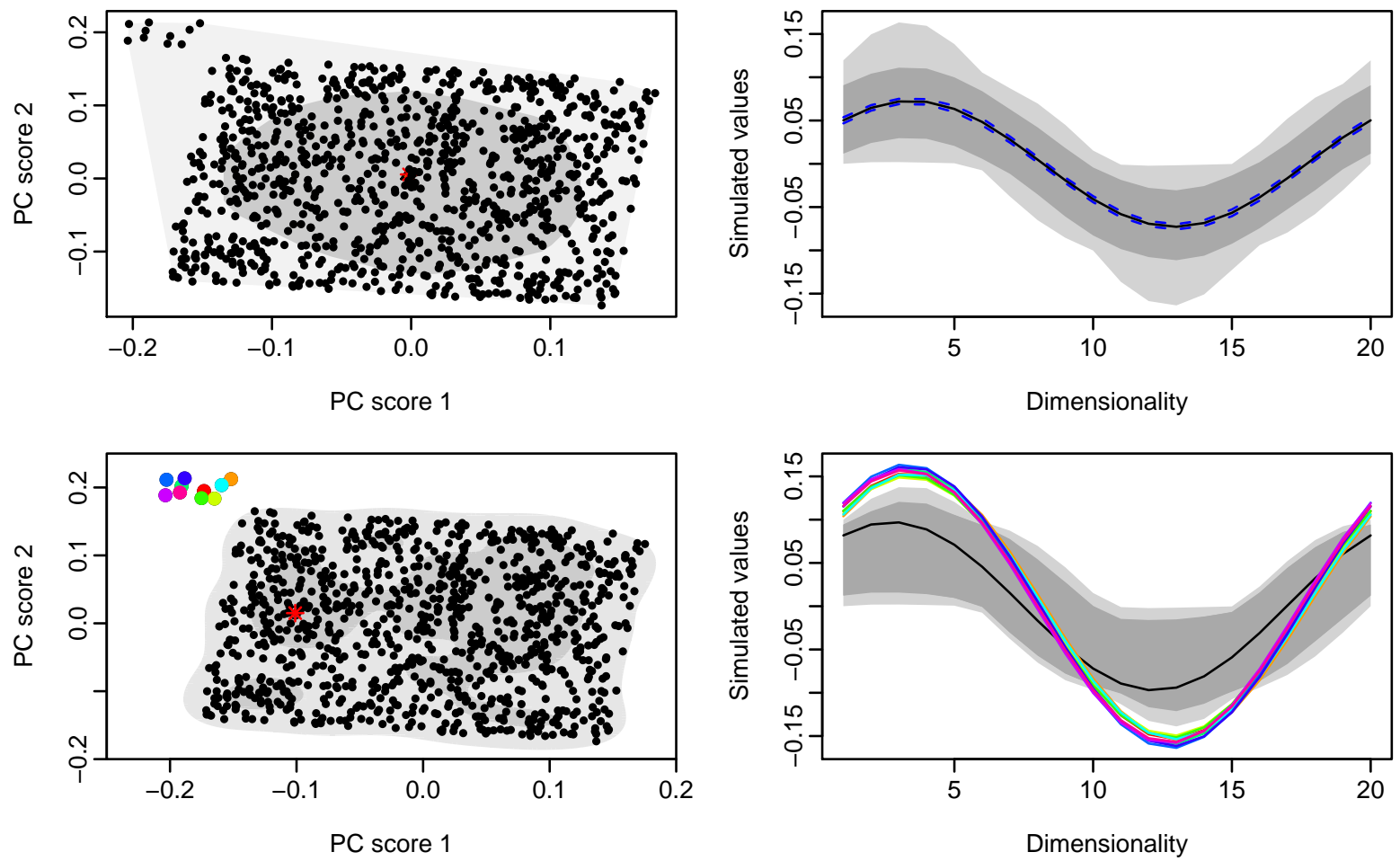

Figure 6: Two proposed graphical outlier detection methods applied to the simulated data set.

\begin{tabular}{lll}
\hline Method & No. detected outliers & Computing time \\
\hline Functional depth & None & 28.5 \\
Integrated squared error & None & 18.82 \\
Functional bagplot & None & 0.56 \\
Functional HDR boxplot & 10 & 0.02 \\
Location-scale & 10 & 0.08 \\
Robust Mahalanobis distance & 1 & 9.72 \\
\hline
\end{tabular}

Table 3: Outlier detection performance and computational speed between two proposed approaches and two existing functional and two high-dimensional multivariate outlier detection methods based on a simulated data set.

With the $99 \%$ coverage probability of the outer region, a bagplot and boxplot for the data are shown in Figure 6. The depth approaches have failed to identify any outliers because the curves are not sufficiently distant from the median. In contrast, the HDR boxplot identifies outliers correctly. 


\section{Discussion and conclusions}

In this paper, we have proposed three graphical tools for visualizing functional data and identifying functional outliers. Ranking principal component scores by data depth or data density is done in a familiar two-dimensional space, from which outliers and inliers are separated. Graphical displays are achieved by matching scores obtained from a bivariate bagplot and a HDR boxplot back to the functional curves.

The advantages of the proposed approaches are that they detect outliers accurately with fast computational speed as well as simultaneously providing a graphical representation. As illustrated by two real data sets, the proposed methods perform better than the existing approaches to outlier detection which either identify spurious outliers or miss obvious outliers.

Through a simulated data set, we have demonstrated that the depth-based methods fail to detect some outliers that are not far from the median curve. In contrast, a density-based approach, such as the HDR boxplot, can identify such outliers correctly.

The methods presented in this paper can be easily extended in two directions. The principal components decomposition that is used in several of our proposed methods could be replaced with other dimension reduction methods such as independent component analysis used in Epifanio (2008) or partial least squares used in Faber et al. (2003). Tukey's halfspace depth may also be replaced by other depth measures if they are more appropriate for capturing a certain aspect of the data. For example, if the underlying distribution is close to elliptical, then it is more efficient to use the Mahalanobis depth approach.

R (R Development Core Team 2008) code for constructing the bagplot, functional bagplot, HDR boxplot and functional HDR boxplot are available upon request from the authors. 


\section{Appendix A. Proof on coverage probability for bagplot}

Because of the affine invariant property of depth, we assume, without loss of generality, a bivariate standard normal distribution with center $O=(0,0)$.

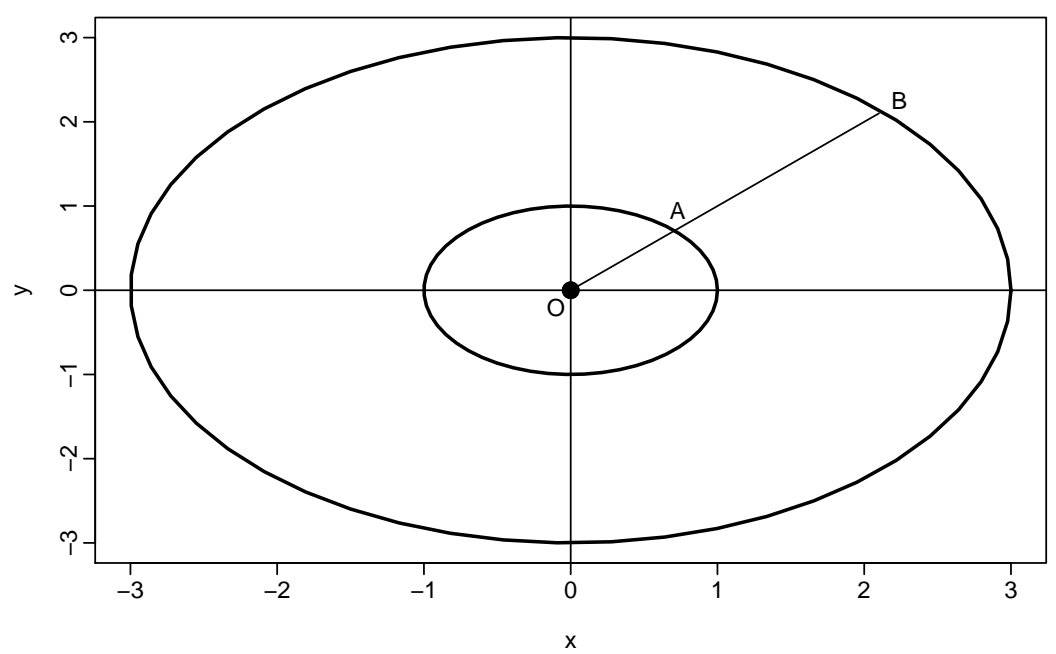

Figure 7: A concept diagram to show the inflation factor in the bagplot.

Let $A$ be a point on the ellipse of a bivariate standard normal distribution associated with a constant probability density contour with coverage 0.5 . Similarly let $B$ be a point on the ellipse associated with a probability density contour with coverage $1-\alpha$. We denote the distances between the origin and $A$ and $B$ as $k_{0.5}$ and $k_{\alpha}$ respectively. Let $\rho_{\alpha}=k_{\alpha} / k_{0.5}$ be the inflation factor used in constructing the fence of the bagplot. Then $\alpha$ is the probability of any point being an outlier when the original data are bivariate normal.

To compute $k_{\alpha}$, we must solve the following bivariate integral,

$$
\iint_{x^{2}+y^{2} \leq k_{\alpha}^{2}} \frac{1}{2 \pi} \exp -\frac{\left(x^{2}+y^{2}\right)}{2} d x d y=1-\alpha .
$$

Switching to polar coordinates, we obtain

$$
\frac{1}{2 \pi} \int_{0}^{k_{\alpha}} \int_{0}^{2 \pi} e^{-r^{2} / 2} r d r d \theta=\frac{1}{2} \int^{k_{\alpha}^{2}} e^{-u / 2} d u
$$


where $u=r^{2}$. The expression (3) under the integral can be immediately recognized as a $\chi^{2}$ density with 2 degrees of freedom. Therefore, $k_{0.5}=1.1774, k_{0.99}=3.0349$ and $\rho_{0.01}=2.5776$. By comparison, $\rho_{0.002}=3$. 


\section{References}

Becker, C. \& Gather, U. (2001), 'The largest nonidentifiable outlier: a comparison of multivariate simultaneous outlier identification rules', Computational Statistics and Data Analysis 36(1), 119-127.

Croux, C. \& Ruiz-Gazen, A. (2005), 'High breakdown estimators for principal components: the projection-pursuit approach revisited', Journal of Multivariate Analysis 95(1), 206-226.

Dioses, T., Dávalos, R. \& Zuzunaga, J. (2002), 'El Niño 1982-1983 and 1997-1998: Effects on Peruvian Jack Mackerel and Peruvian Chub Mackerel', Investigaciones marinas 30(1), 185-187.

Duong, T. \& Hazelton, M. L. (2005), 'Cross-validation bandwidth matrices for multivariate kernel density estimation', Scandinavian Journal of Statistics 32(3), 485-506.

Epifanio, I. (2008), 'Shape descriptors for classification of functional data', Technometrics 50(3), 284-294.

Faber, N., Song, X. \& Hopke, P. (2003), 'Sample-specific standard error of prediction for partial least squares regression', TrAC Trends in Analytical Chemistry 22(5), 330-334.

Febrero, M., Galeano, P. \& Gonzalez-Manteiga, W. (2007), 'A functional analysis of NOx levels: location and scale estimation and outlier detection', Computational Statistics 22(3), 411-427.

Febrero, M., Galeano, P. \& González-Manteiga, W. (2008), 'Outlier detection in functional data by depth measures, with application to identify abnormal NOx levels', Environmetrics 19(4), 331345.

Ferraty, F. \& Vieu, P. (2006), Nonparametric functional data analysis: theory and practice, Springer, New York. 
Filzmoser, P. (2004), A multivariate outlier detection method, in S. Aivazian, P. Filzmoser \& Y. Kharin, eds, 'Proceedings of the Seventh International Conference on Computer Data Analysis and Modeling', Vol. 1, Belarusian State University, Minsk, pp. 18-22.

Filzmoser, P., Maronna, R. \& Werner, M. (2008), 'Outlier identification in high dimensions', Computational Statistics \& Data Analysis 52(3), 1694-1711.

Hardin, J. \& Rocke, D. M. (2005), 'The distribution of robust distances', Journal of Computational and Graphical Statistics 14(4), 928-946.

Human Mortality Database (2008), University of California, Berkeley (USA), and Max Planck Institute for Demographic Research (Germany). Viewed 15/4/07, available online at $<$ www.mortality.org $>$ or $<$ www.humanmortality.de $>$.

Hyde, V., Jank, W. \& Shmueli, G. (2006), 'Investigating concurrency in online auctions through visualization', The American Statistician 60(3), 241-250.

Hyndman, R. J. (1996), 'Computing and graphing highest density regions', The American Statistician 50(2), 120-126.

Hyndman, R. J. \& Ullah, M. S. (2007), 'Robust forecasting of mortality and fertility rates: A functional data approach', Computational Statistics \& Data Analysis 51(10), 4942-4956.

Jones, M. C. \& Rice, J. A. (1992), 'Displaying the important features of large collections of similar curves', The American Statistician 46(2), 140-145.

Kargin, V. \& Onatski, A. (2008), 'Curve forecasting by functional autoregression', Journal of Multivariate Analysis 99(10), 2508-2526.

Liu, R. Y., Parelius, J. M. \& Singh, K. (1999), 'Multivariate analysis by data depth: Descriptive statistics, graphics and inference (with discussion)', The Annals of Statistics 27(3), 783-858. 
Locantore, N., Marron, J. S., Simpson, D. G., Tripoli, N., Zhang, J. T. \& Cohen, K. L. (1999), 'Robust principal component analysis for functional data', Test 8(1), 1-73.

López-Pintado, S. \& Romo, J. (2006), On the concept of depth for functional data, Technical Report 06-30, Departamento de Estadística, Universidad Carlos III de Madrid.

URL: http://docubib.uc3m.es/WORKINGPAPERS/WS/ws063012.pdf

Moran, E. F., Adams, R., Bakoyéma, B., Stefano, F. T. \& Boucek, B. (2006), 'Human strategies for coping with El Niño related drought in Amazônia', Climatic Change 77, 343-361.

R Development Core Team (2008), R: A Language and Environment for Statistical Computing, R Foundation for Statistical Computing, Vienna, Austria. ISBN 3-900051-07-0.

URL: http://www.R-project.org

Ramsay, J. O. \& Ramsey, J. B. (2002), 'Functional data analysis of the dynamics of the monthly index of nondurable goods production', Journal of Econometrics 107(1-2), 327-344.

Ramsay, J. O. \& Silverman, B. W. (2002), Applied Functional Data Analysis: methods and case studies, Springer, New York; London.

Ramsay, J. O. \& Silverman, B. W. (2005), Functional Data Analysis, 2nd edn, Springer, New York.

Reiss, P. T. \& Ogden, T. R. (2007), 'Functional principal component regression and functional partial least squares', Journal of the American Statistical Association 102(479), 984-996.

Rocke, D. M. (1996), 'Robustness properties of S-estimators of multivariate location and shape in high dimension', The Annals of Statistics 24(3), 1327-1345.

Rousseeuw, P. J. \& Ruts, I. (1998), 'Constructing the bivariate Tukey median', Statistica Sinica 8, 827-839.

Rousseeuw, P. J. \& Ruts, I. (1999), 'The depth function of a population distribution', Metrika 49(3), 213-244. 
Rousseeuw, P., Ruts, I. \& Tukey, J. W. (1999), 'The bagplot: A bivariate boxplot', The American Statistician 53(4), 382-387.

Scott, D. W. (1992), Multivariate density estimation: theory, practice, and visualization, Wiley, New York.

Sood, A., James, G. M. \& Tellis, G. J. (2009), 'Functional regression: a new model for predicting market penetration of new products', Marketing Science . forthcoming.

Struyf, A. \& Rousseeuw, P. J. (2000), 'High-dimensional computation of the deepest location', Computational Statistics \& Data Analysis 34(4), 415-426.

Timmermann, A., Oberhuber, J., Bacher, A., Esch, M., Latif, M. \& Roeckner, E. (1999), 'Increased El Niño frequency in a climate model forced by future greenhouse warming', Nature 398(6729), 694-697.

Tukey, J. W. (1974), Mathematics and the picturing of data, in 'Proceedings of the International Congress of Mathematicians', Vancouver, pp. 523-531.

Tukey, J. W. (1977), Exploratory Data Analysis, Addison-Wesley, London.

Wang, J. \& Serfling, R. J. (2006), Data depth: robust multivariate analysis, computational geometry and applications, in R. Y. Liu, R. J. Serfling \& D. L. Souvaine, eds, 'On scale curves for nonparametric description of dispersion', American Mathematical Society, pp. 37-48.

Zhang, L., Marron, J. S., Shen, H. \& Zhu, Z. (2007), 'Singular value decomposition and its visualization', Journal of Computational and Graphical Statistics 16(4), 833-854.

Zuo, Y. \& Serfling, R. J. (2000), 'General notions of statistical depth function', The Annals of Statistics 28(2), 461-482. 